\title{
Absorption Spectra of Lanthanide and Actinide lons
}

\author{
Jørgensen, Christian Klixbüll
}

Published in:

Journal of Chemical Physics

Link to article, DOI:

$10.1063 / 1.1741980$

Publication date:

1954

Document Version

Publisher's PDF, also known as Version of record

Link back to DTU Orbit

Citation (APA):

Jørgensen, C. K. (1954). Absorption Spectra of Lanthanide and Actinide lons. Journal of Chemical Physics, 23(2), 399-400. https://doi.org/10.1063/1.1741980

\section{General rights}

Copyright and moral rights for the publications made accessible in the public portal are retained by the authors and/or other copyright owners and it is a condition of accessing publications that users recognise and abide by the legal requirements associated with these rights.

- Users may download and print one copy of any publication from the public portal for the purpose of private study or research.

- You may not further distribute the material or use it for any profit-making activity or commercial gain

- You may freely distribute the URL identifying the publication in the public portal

If you believe that this document breaches copyright please contact us providing details, and we will remove access to the work immediately and investigate your claim 
The variation of $C$ with $\Delta$ gives a more direct indication of the effect of exchange on $C$ than the variation of $C$ with concentration, since the former is less affected by inhomogeneities in the solid solution than the latter. This assertion is supported by the fact that the points in plots of $C$ versus $\Delta$ scatter much less than the points in plots of $C$ versus concentration.

Several published results have been excluded from our comparison because their points of $C / C_{c}$ versus $\Delta / \Delta_{c}$ scatter excessively.

The comparison of five studies on four systems does not justify the drawing of detailed conclusions. The following does, however, appear: (1) It is in general not correct to assume that $C$ does not depend on exchange. (2) It seems that $C$ is nearly independent of exchange when the orbital angular momentum of the paramagnetic ion is only slightly quenched (as in $\mathrm{Pu}^{+4}$ ) or when it is nearly zero for the free ion (as in $\mathrm{Mn}^{++}$).

1 J. H. Van Vleck, Electric and Magretic Susceptibilities (Oxford Uthiversity Press, New York, 1932).

2W. G. Penney and R. Schlapp, Phys. Rev. 41, 194 (1932) and R. Schlapp and $W . G$. Penney, Phys. Rev. 42, $666(1932)$. Rev. 79, 705 (1950); and J.S. Smart, Phys. Rev. 86, 968 (1952).

\section{Errata: Absolute Quantum Efficiencies of Luminescence of Organic Molecules in Solid Solution \\ [J. Chem. Phys. 20, 829 (1952)] \\ Earl H. Gilmore* George E. Gibson, and Donald S. McClure Depariment of Chemistry and Chemical Engineering, University of California, Berkeley, California}

$\mathrm{T}$ $\mathrm{HE}$ correction for the ratio of the apparent solid angle to the actual solid angle due to the passage of the luminescence emission from a denser to a rarer optical medium was incorrectly calculated in the above-named article. This correction factor was given for a $45^{\circ}$ angle of observation of the specimens as $\left(2 n^{2}-1\right)^{-1}$ in which $n$ is the refractive index in the denser medium referred to air as unity, which is the rarer medium.

A reconsideration of the geometry of the situation shows that this correction factor for an arbitrary value of the angle of observation, $\phi$, from the normal should be $I(\phi, \nu)=I_{\nu} n^{-1} \cos \phi$ $\left(n^{2}-\sin ^{2} \phi\right)^{-1}$ which gives $n^{-1}\left(2 n^{2}-1\right)^{-1}$ for $\phi=\pi / 4$.

Such a correction will have the effect of increasing the quantum yields of the various luminescences listed in Table $I$, page 835 by the following factors: benzophenone, 1.43; acetophenone, 1.44; acetone, 1.43 ; benzene phosphorescence, 1.44-fluorescence, 1.47; fluorobenzene, 1.47; chlorobenzene, 1.43; bromobenzene, 1.42; naphthalene phosphorescence, 1.42-fluorescence, 1.45 ; triphenylene phosphorescence, 1.42-fluorescence, 1.44. The error has an insignificant effect on the quantities listed in Table III. The corrected lifetime in Table IV should read as follows:

$\begin{array}{lc}\text { Compound } & \text { Corrected lifetime } \\ \text { Benzophenone } & \text { sec } \\ \text { Acetophenone } & 0.008 \\ \text { Acetone } & 0.013 \\ \text { Benzene } & 0.014 \\ \text { Chlorobenzene } & 21.0 \\ \text { Naphthalene } & 0.007 \\ \text { Triphenylene } & 11.0 \\ \end{array}$

It is to be noted that, with this correction, our quantum yield values for the fluorescence of both benzene and naphthlene are considerably greater than those obtained by Bowen and Williams for liquid solutions. ${ }^{1}$

Several workers who measured fluorescence yield values for solutions of sodium fluorescein have not included this correction. ${ }^{2-4}$ For observations along the direction normal to the fluorescing surface this correction requires that their quantum yield values be multiplied by $n^{2}$ (about 1.75 for water). Their quantum yield values would thereby be raised considerably above unity. It is not clear just what compensating error may have reduced their values to below unity, but it seems that any analysis of the optical problem involved must include in addition to reabsorption corrections, corrections for fluorescence resulting from the internal absorption of primary fluorescence. Because absorption and fluorescence bands do not overlap significantly in rigid glass solutions at $77^{\circ} \mathrm{K}$, our experiments were free of this complication. One of us is presently engaged in the effort to estimate the magnitudes of these secondary effects for systems in which they can be expected. The quantum values of Umberger and $\mathrm{La} \mathrm{Mer}{ }^{6}$ and of Forster and Livingston, ${ }^{B}$ do not require a correction for the refraction effect.

We acknowledge also Dr. W. Metcalf's recognition of the error in our article although one of us had become aware of it before his communication arrived.

* Now at Department of Chemistry, Oklahoma Agricultural and Mechanical College, Stillwater, Oklahoma.

1 E. J. Bowen and A. H. Williams, Trans, Faraday Soc, 35, 765 (1939). 2 S. I. Vavilov, Z. Physik 22, 266 (1924).

${ }^{3}$ H. Hellstrom, Arkiv Kemi, Mineral Geol. 12A, 17 (1937)

I. C. Ghosh and S. B. Sen-Gupta, Z. physik. Chem, B41, 117 (1938).

$5 \mathrm{~J}$. Umberger and V. K. La Mer, J. Am. Chem. Soc. $67,1099(1945)$
L. S. Forster and R. Livingston, J. Chem. Phys, 20, 1315 (1952).

\section{Absorption Spectra of Lanthanide and Actinide Ions}

Chr. Klixbúll Jórgensen

Chemistry Department A, Technical Universtity of Denmark, Copenhagen, Denmars

(Received August 16, 1954)

$\mathrm{T}$

HE narrow absorption bands of lanthanide and actinide ions are due to transitions between states which are equivalent to the states of the free ion in vacuo. In the case of two effective $f$-electrons as exemplified by trivalent praseodymium ${ }^{1}$ and thulium $^{2}$ it has been possible to identify the transitions and assign quantum numbers to the excited states, while the ground state is invariable. Recently, Satten ${ }^{3}$ interpreted the neodymium spectrum and applied Condon and Shortley's theory to this $f^{3}$ system. The ratio between the parameters of electrostatic interaction $F_{6} / F_{2}$ is erroneously given ${ }^{3}$ to be $<0.00306$, while the condition really is $<0.0306$. If $F_{6}$ is given not negligible values, the weak bands of $\mathrm{Nd}^{+++}$seem better in accordance with theory, while the strong bands still are identified as the quartet-transitions. If $F_{8}=0.02 F_{2}$ and $F_{4}=0.2 F_{2}$, it is nearly certain that the weak band groups at 6250,4710 , and $4620 \mathrm{~A}$ correspond to transitions to ${ }^{2} \mathrm{H}$, ${ }^{2} \mathrm{G}$, and ${ }^{2} \mathrm{~K}$, respectively. The single band at $4275 \mathrm{~A}$ is presumably due to ${ }^{2} P_{1}$.

In the actinides, Gruen ${ }^{5}$ has extrapolated the Condon-Shortley parameters for $\mathrm{NpO}_{2}^{+}$and $\mathrm{PuO}_{2}{ }^{++}$from the $f^{2}$ system $\mathrm{Th}^{++}$, known from atomic spectroscopy. ${ }^{6,7}$ It can be questioned if the electrostatic interaction is proportional to the atomic number minus a large shielding constant ( 34 in the lanthanides ${ }^{3}$ and 58 in the actinide ions ${ }^{5}$ ). Thus, ${ }_{1}^{1} F_{2}$ is $232 \mathrm{~cm}^{-1}$ in $\mathrm{Pr}^{+++}$, but only 93 $\mathrm{cm}^{-1}$ in $\mathrm{La}^{+}$. The isoelectronic species $\mathrm{Np}^{+++}$and $\mathrm{Pu}^{+4}$ have absorption bands ${ }^{8}$ which are nearly biuniquely corresponding, with the wave numbers 19 percent higher in $\mathrm{Pu}^{+4}$ than in $\mathrm{Np}^{+3}$. The strong-shielding theory would give only three percent difference, while of course the increased Lande factor $\xi$ is also important for the evolution of intermediate coupling. The electrostatic interaction seems nevertheless to be roughly proportional to $z+1$, where $z$ is the external charge of the ion. This can be connected with the behaviour of the outer electrons, i.e., the ionic radius, which is strongly dependent of $z$.

The absorption spectra of the actinide ions can also be used for estimate of the energy difference between $5 f$ - and $6 d$-electrons. Transitions $f^{n} \rightarrow f^{n-1} d$ are not forbidden as free dipole radiation and have correspondingly high intensity as found in the ultraviolet bands of $\mathrm{Ce}^{+++}$. These bands must of course not be confused with the molecular spectra, due to internal electron transfer, found e.g. in the chlorocomplexes of $\mathrm{U}^{+4}, \mathrm{Pu}^{+4}, \mathrm{Fe}^{+3}, \mathrm{Cu}^{+2}, \mathrm{Ir}^{+4}$ 
with decreasing wave number by increasing ease of the redox process to the next lower oxidation state. The actinide hypothesis of Seaborg ${ }^{8}$ seems to be strongly supported by the absence of such strong bands in uranium (IV) aquo ions over $2400 \mathrm{~A}$. Papers on the spectra of $\mathrm{U}^{+3}, \mathrm{U}^{+4}$ and other actinide ions will be published elsewhere. The strong deviations from Russell-Saunders coupling intermix in some cases the quantum states with same $J$ completely. This may explain anomalous intensity relations, as found in $\mathrm{Am}^{+++}$. The interaction between different electron configuration causes slight deviations from the Condon-Shortley theory.

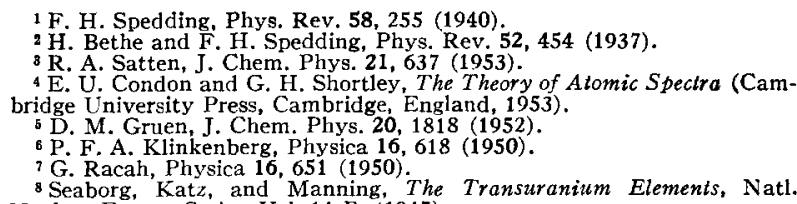

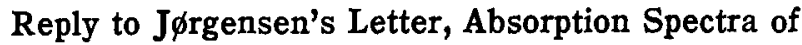 Lanthanite and Actinide Ions}

ROBERI A. SATTEN

University of California, Los Angeles, California

(Received October 13, 1954)

$\mathbf{J}^{\rho}$

RGENSEN is correct in pointing out in the accompanying letter ${ }^{1}$ that the radial integrals $F_{6}$ and $F_{2}$ satisfy $F_{6} / F_{2}<0.0306$ instead of $<0.00306$ as reported. ${ }^{2}$ However, the ratio was correctly calculated and reported in the form $F_{6} / F_{2}<225 / 7361.64$, the error being made in the placement of the decimal point upon division. Furthermore, no conclusion of the paper is affected. The only use made of this inequality was, along with the one for $F_{4} / F_{2}$, to show that the set of $F_{k}$ 's used by Lange for the $\mathrm{Pr}^{+++}$spectrum must be incorrect. This conclusion is still true since Lange's assignments lead to a value of 0.606 for the ratio $F_{4} / F_{2}$, which is about three times too large. This ratio is the one to which the energy calculations are the most sensitive.

The values of the $F_{k}$ ratios which I used for the calculations of the doublet levels were chosen to agree with the assignments of Condon and Shortley for the $4 f^{2}$ configuration of the La II spectrum. These ratios were also used by Spedding ${ }^{5}$ for the $\mathrm{Pr}^{+++}$ spectrum. Although it was realized and stated at the time that the $F_{4} / F_{2}$ ratio of Condon and Shortley was too large, it was regarded as too uncertain a procedure at this stage to modify it or to attempt to adjust the $F_{k}$ integrals to agree with doublet assignments to the $\mathrm{Nd}^{+++}$spectrum, for this adjustment can be made in more than one way within the limits of accuracy imposed by the use of a Russell-Saunders model for the Nd ion. One can expect the Russell-Saunders model to be only a rough approximation because of the relatively strong spin-orbit interaction as well as because of the effect of the strong crystalline electric field on those $4 f^{3}$ free ion states which are very close together. The influence of the latter in a particular case is discussed in the next to last paragraph of this letter.

To attempt to adjust $F_{k}$ values or ratios on the basis of an empirical fit to the doublet states of the $\mathrm{Nd}^{+++}$spectrum when the quantum number assignments are largely uncertain is thus not easy to do correctly. The quartet states, however, satisfy relations which are independent of the $F_{k}$ values so that in these assignments, or at least in some of them, one can hope to be correct. It is for this reason that no doublet assignments were made except in one case $\left({ }^{2} D_{1}\right)$ where an observed level occured in the midst of a wide region in which no quartet levels were predicted. Even this doublet assignment is questionable.

One can definitely say, however, that the $F_{4} / F_{2}$ ratio will not equal its limiting value as assumed by J $\not$ rgensen, since this equality would imply that the square of the radial part of the $4 f$ one-electron wave function is a delta function, which of course is very far from the case. The greater the spread of the $4 f$ radial wave function for a given position of its maximum value (roughly) the smaller the $F_{k} / F_{k},\left(k^{\prime}>k\right)$ ratios will be. Furthermore, it is impossible, as J $\varnothing$ rgensen does, to choose the limiting value of $F_{4} / F_{2}$ without at the same time choosing the limiting value of $F_{6} / F_{2}$. Thus while Jørgensen may turn out to be correct or partially correct in interpreting the 6250,4710 , and $4620 \mathrm{~A}$ groups as ${ }^{2} \mathrm{H}$, ${ }^{2} G$, and ${ }^{2} K$, there seems to be at present no compelling reason to single out these particular interpretations.

The case of the $4275 \mathrm{~A}$ group involving only a single upper level is probably an example of a ${ }^{2} P_{1}$ level, as Jørgensen says, strongly perturbed by some other state. ${ }^{2}$ The perturbation is most likely to be the result of the interaction with another of the $4 f^{3}$ states of the free ion which is very close to the ${ }^{2} P_{3}$ level. This perturbation is a result of the mixing of states due to the strong crystalline electric field. The evidence in part for this explanation is that the 4275 A group has a higher energy than can result from any assignment of the $F_{k}$ or $\zeta$ integrals even in intermediate coupling as the spin-orbit interaction would not be nearly large enough even if in the right direction and configuration interaction would most likely push the level to even lower energies. In agreement with the latter we note that the sharp absorption line at $4273 \mathrm{~A}$ is so sharp even at room temperatures that it is very unlikely that much contribution to the wave function of its upper level comes from outer electronic configurations. If this argument is a valid one, the transition is probably largely magnetic dipole and the perturbing state is ${ }^{2} G_{9 / 2}$.

I should also like to point out that in reference 2 there is an obvious misprint in Table I. The expression for ${ }^{2} L$ should begin with $3 F_{0}$ and not $3 F_{2}$. The $F_{2}$ term is absent.

$$
\begin{aligned}
& 1 \text { C. K. Jørgensen, J. Chem. Phys., 23, } 399(1955) \text {. } \\
& 2 \text { R. A. Satten, J. Chem. Phys. 21, 637 (1953). } \\
& 3 \text { H. Lange, Ann. Physik 31, 609 (1938). } \\
& \text { E. U. Condon and G. H. Shortley, The Theory of Atomic Spectra (Cam- } \\
& \text { bridge University Press, Cambridge. England). }
\end{aligned}
$$

6 F. Spedding, Phys. Rev, 58, 255 (1940).

\section{Excitation of Molecules in the Beta Decay of a Constituent Atom*}

H. M. Schwartz

Depariment of Physics, Universily of Arkansas, Fayelleville, Arkansas (Received November 1, 1954)

A TOMIC excitation in beta decay has been studied theo$A$ retically. ${ }^{1}$ This report summarizes initial results for the corresponding molecular problem. ${ }^{2}$

By sudden perturbation theory, the probability, $P_{A B}$, for a molecular transition $A \rightarrow B$ to accompany a radioactive process within the molecule, is

$$
\left|\int \psi_{B}^{(2) *} \exp \left(i \mathbf{q} \cdot \mathbf{x}_{1} / \hbar\right) \psi_{A}^{(1)} d \tau\right|^{2}
$$

where $\mathbf{x}_{1}=$ position vector of decaying nucleus and $\mathbf{q}=$ its recoil momentum. With the Born-Oppenheimer approximation for the $\psi$ and a Franck-Condon type of evaluation, this is

$$
\begin{gathered}
P_{A B}=P_{\alpha \beta} P_{a b}, \quad P_{\alpha \beta}=\left|\int \varphi \beta^{(2) *}(\xi ; x) \varphi_{\alpha}^{(1)}(\xi ; \bar{x}) d \xi\right|^{2} \\
P_{a b} \equiv P_{a b}(\alpha, \beta ; \mathbf{q})=\left|\int \chi b, \beta^{(2) *}(x) \exp \left(i \mathbf{q} \cdot \mathbf{x}_{1} / h\right) \chi_{a, \alpha^{(1)}}(x) d x\right|^{2}
\end{gathered}
$$

where $\xi, x$ symbolize the set of electronic and nuclear coordinates respectively. The completeness theorem for the $\chi$ yields $\Sigma_{b} P_{a b}=1$ with the consequent obvious interpretation of the $P_{\alpha \beta}$ and $P_{a b}$ as absolute and relative probabilities respectively.

For polyatomic molecules the $P_{a b}$ can be evaluated readily in the approximation of separated vibrational motion under Hooke's law forces, when the normal coordinates are known. ${ }^{3}$ Comparison of Eq. (2) and the vibrational equation solution, ${ }^{3}$ shows that $P_{a b}$ is a product involving for each degree of freedom an integral of the same type as occurs in the diatomic case, discussed below. 